\title{
Anthropometric Measures and Breast Cancer Risk among Hispanic Women in Puerto Rico
}

\section{Farah A Ramírez-Marrero ( $\nabla$ farah.ramirez1@upr.edu )}

University of Puerto Rico Rio Piedras: Universidad de Puerto Rico Recinto de Rio Piedras https://orcid.org/0000-0003-00030683

\section{Cruz Maria Nazario}

University of Puerto Rico Medical Sciences Campus: Universidad de Puerto Rico Recinto de Ciencias Medicas

\section{Rosa V Rosario-Rosado}

University of Puerto Rico Medical Sciences Campus

\section{Michelle Schelske-Santos}

University of Puerto Rico Rio Piedras: Universidad de Puerto Rico Recinto de Rio Piedras

Imar Mansilla-Rivera

University of Puerto Rico Medical Sciences Campus: Universidad de Puerto Rico Recinto de Ciencias Medicas

Jing Nie

Buffalo State The State University of New York: SUNY Buffalo State College

\section{Johan M Hernandez-Santiago}

University of Puerto Rico Medical Sciences Campus: Universidad de Puerto Rico Recinto de Ciencias Medicas

\section{Jo Freudenheim}

Buffalo State The State University of New York: SUNY Buffalo State College

\section{Research Article}

Keywords: Body size, waist to height ratio, adiposity, breast cancer, Hispanic women

Posted Date: October 28th, 2021

DOI: https://doi.org/10.21203/rs.3.rs-1021850/v1

License: (1) This work is licensed under a Creative Commons Attribution 4.0 International License. Read Full License 


\section{Abstract}

While there is consistent evidence of increased risk of postmenopausal breast cancer associated with higher body mass index (BMI), higher adult weight gain and higher waist circumference in North American and European populations, there is little evidence for Hispanic women. Among Hispanic women in Puerto Rico (PR), breast cancer is the leading type of cancer, and leading cause of cancer related deaths. However, compared with the United States, breast cancer rates are lower but increasing more rapidly. Purpose: To determine associations between anthropometric characteristics and breast cancer risk in Hispanic women in PR. Methods: Data from a population-based case control study in the San Juan metropolitan region was used to examine associations between anthropometric measures and breast cancer risk, also considering menopausal status and hormone therapy (HT). Results: Among premenopausal women, BMl equal or higher than $25 \mathrm{~kg} / \mathrm{m}^{2}$ and waist to height ratio (WHtR) of 0.53 or higher were associated with lower odds of breast cancer. For postmenopausal breast cancer, waist circumference of $86.4 \mathrm{~cm}$ or higher, WHtR of 0.57 or higher, waist to hip ratio of 0.84 or greater, and height of $150 \mathrm{~cm}$ or taller were associated with lower odds of breast cancer. Conclusion: Our study provides evidence that associations of risk with anthropometry may differ for Hispanic women. Future studies should include measures of fat and lean mass distribution to further understand anthropometric measures and breast cancer risk among Hispanic women.

\section{Introduction}

Human body size and proportions are practical and feasible measures historically associated with health and disease. For example, height and weight are basic anthropometric measures used to identify people in different body mass index (BMI = weight in $\mathrm{kg}$ divided by height in $\mathrm{m}^{2}$ ) categories associated with increased risk of health complications and premature mortality [1]. Adults with a BMI between 18.5 to $24.9 \mathrm{~kg} / \mathrm{m}^{2}$ are typically classified as "normal" or "healthy", those within 25.0 to $29.9 \mathrm{~kg} / \mathrm{m}^{2}$ as "overweight", and those at or above $30 \mathrm{~kg} / \mathrm{m}^{2}$ as "obese"; with the latter particularly associated with a higher mortality risk from all causes ${ }^{1}$.

Although variation in body weight is likely to be influenced by excessive adiposity or fat accumulation, other factors such as body water and lean mass (muscle, tendons, and bone) also influence body weight and BMI. Therefore, the use of BMI as a screening tool for "adiposity-based chronic disease" or ABCD as recently presented by the American Association of Clinical Endocrinologists and the American College of Endocrinology [2], is intrinsically problematic because it does not account for differences in proportions of adipose tissue and lean body mass. There is consistent evidence of excess central adiposity with inflammation, insulin resistance, and risk of many chronic diseases; but excess adiposity in the gluteo-femoral area appears to be protective for cardiometabolic risk [3].

Another problem with BMI as a screening tool is potential inaccuracies of the established cut points among people of different ethnicities. It has also been suggested that BMI cut points should not be universally applied, and that population specific criteria must be identified [4]. For example, Deurenberg-Yap et al. [5] reported cases of low BMI but high body fat among people living in Singapore and recommended a different BMI cut point for obesity classification $\left(\geq 27 \mathrm{~kg} / \mathrm{m}^{2}\right)$. Later, the World Health Organization [6] suggested that BMI cut points for Asian populations should be different from standard values (overweight $=25$ to $27.7 \mathrm{~kg} / \mathrm{m}^{2}$ ). Rush et al. [7] also observed ethnic differences among women from South Africa, Europe, and New Zealand in the relationship between BMI and body fat that were explained by differences in central adiposity and muscle mass. Analyses of BMI and health outcomes are based on meta-analyses and population-based studies mostly from North America, Western Europe, Australia, Japan, and China, with infrequent inclusion of Hispanic groups. Herrera et al. [8] used data from the Latin-American Consortium of Studies in Obesity to determine cut points for BMI, waist circumference, and waist to hip ratio (WHR) in screening for coronary heart disease risk. Although the authors did not recommend changes in BMI cut points for obesity classification, they deemed BMI as almost uninformative in that population and the ratio between waist and hip circumference as the most accurate anthropometric indicator of coronary heart disease risk.

Excess weight gain and body fat accumulation during adulthood have been consistently associated with increased risk and mortality from breast cancer in postmenopausal women [9-13]. Among premenopausal women, adiposity has been

Page 2/17 
equivocally associated with decreased risk of breast cancer [13-14]. There may be differences by body fat distribution. For example, a case-control study by Godinho-Mota et al. [15] showed that high abdominal adiposity measured by dual-energy Xray absorptiometry (DEXA) increased the risk of developing breast cancer in premenopausal women.

Inconsistent associations between excess fat accumulation, obesity, and breast cancer risk have been reported for different racial/ethnic groups, particularly among Hispanic women. For example, Wenten et al. [16] conducted a population-based casecontrol study in New Mexico and reported that adult weight gain and obesity were risk factors for breast cancer in both Hispanic and non-Hispanic women; different from non-Hispanic white women, the risk was not influenced by menopausal status in the Hispanic group. In another case-control study by Slattery et al. [17], adult weight gain and obesity were associated with increased breast cancer risk among non-Hispanic white women, but with reduced risk among Hispanic women, suggesting that the consequences of obesity on breast cancer risk may differ by race/ethnicity. Most of these studies used BMI to classify participants as overweight or obese [4-5, 7].

Other anthropometric measures that have been related to obesity and breast cancer risk in pre- and postmenopausal women include waist circumference and WHR. Waist circumference is used as an index of body fat accumulation in the abdominal and visceral area associated with elevated insulin resistance, inflammation, and breast cancer risk [18]. Waist circumference at or above $88 \mathrm{~cm}$ in women is considered an indicator of risk equivalent to $30 \mathrm{~kg} / \mathrm{m}^{2}$ of BMI ${ }^{10}$. A WHR at or above 0.85 is another index of abdominal fat accumulation associated with breast cancer risk among both pre- and postmenopausal women [19-20]. However, it has been suggested that the association between waist circumference, WHR, and breast cancer risk may result from their correlation with BMI [21]. There is also evidence suggesting that, like BMI, the relationship between waist circumference and disease risk varies among different populations [22-24]. To our knowledge, there is no consensus regarding anthropometric indicators of obesity as predictors of breast cancer among different race/ethnicity groups.

There are few studies regarding anthropometric characteristics and their association with breast cancer risk among Hispanic women outside the United States (USA) mainland living in their country of origin. In Puerto Rico (PR), cancer is the leading cause of death, and breast cancer is the leading type of cancer and leading cause of cancer deaths among women [25]. Although the rate of breast cancer is lower in PR than in the USA, it is increasing more rapidly [26]. Understanding if breast cancer risk is associated with body size and proportions among Hispanic women in PR is highly relevant. In this study we used data from a population-based case control study of breast cancer in PR to examine associations between anthropometric measures and breast cancer risk.

\section{Materials And Methods}

Recruited were women between 30 and 79 years of age who were residents of the metropolitan area of San Juan to participate in a population-based case control-study (ATABEY Study). The design and implementation have been described in detail elsewhere [27]. The study was approved by the University of Puerto Rico Medical Sciences Campus Institutional Review Board and by the IRB of all participating institutions.

Anthropometric measures were obtained by trained study personnel, and interview data were collected with structured interviews by trained study personnel. Standing and sitting height were measured in $\mathrm{cm}$ with a SECA stadiometer (model 217; SECA North America, California, USA). Standing height was measured without shoes and socks and with light clothing, back and buttocks touching the scale and eyes directed to a perpendicular point in front. Sitting height was measured similar to standing height but with participants sitting in a stool of known height. Sitting height to height ratio was later calculated. Body weight was measured to the nearest $0.1 \mathrm{~kg}$ using a TANITA balance (model BF-522W; TANITA Corporation, Illinois, USA). Participants were asked to remove shoes and socks, clean both feet with an alcohol swab, and stand on the balance with feet touching the metal surface and the scale display showed a zero. BMI was later determined from standing height and weight measures (weigh in $\mathrm{kg} /$ height in $\mathrm{m}^{2}$ ).

Waist circumference was measured in the horizontal plane midway between the lowest rib and the iliac crest after a normal exhalation. Hip circumference was measured in the horizontal plane at the widest part of the buttocks close to the inguinal 
area. Both circumferences were measured to the nearest $0.1 \mathrm{~cm}$ with a Gulick-II anthropometric tape (Performance Health, Chicago, ILL). WHR (waist circumference divided by hip circumference in $\mathrm{cm}$ ) and waist to height ratio (WHtR, waist circumference divided by height in $\mathrm{cm}$ ) were later calculated.

Measures of skinfold thickness in $\mathrm{mm}$, an estimate of subcutaneous adipose tissue and body fat, were obtained at the triceps (vertical fold midpoint between the acromion and olecranon processes), suprailiac (diagonal fold just above the iliac crest and aligned with mid-axillary line), and thigh (vertical fold midpoint between the upper top of the patella and the inguinal crease) using a Lange caliper (Beta Technology, Houston, TX). The sum of these skinfolds was used to estimate body density and percent fat using available equations specifically for Hispanic groups [28].

Body Density $=1.0994921-(0.0009929 \times$ sum of triceps, thigh, and suprailiac skinfolds $)-(0.0001392 \times$ age in years $)$

Body Fat Percentage $=(4.87 /$ body density $)-4.41$

Height, weight, and waist circumference were then used to determine a body shape index (ABSI), a measure developed by Krakauer and Krakauer [29] strongly linked to mortality risk in women [30], and strongly associated with cardiovascular and cancer mortality [31]. Lifetime weight gain was determined from current measured weight minus reported weight at 20 years of age.

All anthropometric measures were taken three times and means reported and compared between cases and controls using the student t-test for continuous and Chi-square tests for categorical variables. Women with missing values were excluded from the specific anthropometric variable related analysis only. For covariates, missing values for age at menarche and age at menopause were replaced with the median by menopausal and case-control status. Spearman correlation coefficients were obtained between all anthropometric variables. Tertiles of each anthropometric variable were created for pre-and postmenopausal women based on the distribution of controls to three groups. Odds ratios and $95 \%$ confidence intervals were computed with unconditional logistic regressions. Odds ratios were adjusted for age, education, age at menarche, parity, age at menopause, previous benign breast disease, and family history of breast cancer. All analyses were examined stratified by menopausal status. Because use of postmenopausal hormone therapy (HT) has been linked to breast cancer risk [32], we also analyzed the risk of postmenopausal breast cancer in our cases and controls stratified by HT. Differences in strata were examined with a multiplicative test of interaction. All statistical tests conducted with the IBM SPSS Statistics for Windows, Version 27.0. (Armonk, NY: IBM Corp), were two-sided and considered statistically significant at an alpha less than 0.05 .

\section{Results}

Characteristics of study participants by case and control status and by menopausal status are shown in Table 1. Compared with controls, cases were somewhat older, among both pre and postmenopausal participants. Regardless of menopausal status, cases had more years of education, fewer births, and more previous benign breast disease than controls. Age at menarche and use of hormonal therapy were not different between post-menopausal cases and controls. 
Table 1

Descriptive Characteristics of Study Participants by Case-Control and Menopausal Status

\begin{tabular}{|c|c|c|c|c|c|c|}
\hline \multirow[t]{3}{*}{ Characteristic } & \multirow{3}{*}{$\begin{array}{l}\text { All } \\
\text { Cases } \\
(n=315)\end{array}$} & \multirow{3}{*}{$\begin{array}{l}\text { All } \\
\text { Controls } \\
(n=348)\end{array}$} & \multicolumn{2}{|c|}{ Pre-menopausal } & \multicolumn{2}{|c|}{ Post-Menopausal } \\
\hline & & & Cases & Controls & Cases & Controls \\
\hline & & & $\begin{array}{l}(n=84 \\
26.7 \%)\end{array}$ & $\begin{array}{l}(n=134, \\
38.5 \%)\end{array}$ & $\begin{array}{l}(n=231 \\
73.3 \%)\end{array}$ & $\begin{array}{l}(n=214 \\
61.5 \%)\end{array}$ \\
\hline Age (years) & $\begin{array}{l}58.7 \\
(11.0)\end{array}$ & $\begin{array}{l}54.0 \\
(13.4)^{\star}\end{array}$ & $45.4(5.3)$ & $40.2(6.4)$ & $63.5(8.2)$ & $62.7(8.5)$ \\
\hline Education (n, \%) & $48,15.2$ & * & $10,11.9$ & * & $38,16.5$ & * \\
\hline$<12$ years schooling & $54,17.1$ & $84,24.1$ & $7,8.3$ & $30,22.4$ & $47,20.3$ & $54,25.2$ \\
\hline 12 years & $84,26.7$ & $69,19.8$ & $24,28.6$ & $25,18.7$ & $60,26.0$ & $44,20.6$ \\
\hline Technical/vocational/associate & $82,26.0$ & $96,27.6$ & $26,31.0$ & $39,29.1$ & $56,24.2$ & $57,26.6$ \\
\hline Bachelor & $47,14.9$ & $77,22.1$ & $17,20.2$ & $28,20.9$ & $30,13.0$ & $49,22.9$ \\
\hline Graduate & & $22,6.3$ & & $12,9.0$ & & $10,4.7$ \\
\hline Age at menarche (years) & $\begin{array}{l}12.2 \\
(1.6)\end{array}$ & $12.5(1.8)$ & $12.0(1.6)$ & $12.5(1.9)$ & $12.3(1.7)$ & $12.5(1.8)$ \\
\hline Number of births & $2.1(1.5)$ & $2.5(1.7)^{\star}$ & $1.7(1.3)$ & $2.3(1.5)^{\star}$ & $2.3(1.6)$ & $2.6(1.7)^{\star}$ \\
\hline Age at menopause (years) & / & / & / & / & $47.4(6.9)$ & $46.8(6.4)$ \\
\hline $\begin{array}{l}\text { Family history of breast cancer ( } \mathrm{n} \text {, } \\
\text { \%yes) }\end{array}$ & $66,21.0$ & $30,8.6^{\star}$ & $11(13.1)$ & $7,5.2^{\star}$ & $55,23.8$ & $23,10.7 *$ \\
\hline $\begin{array}{l}\text { Previous benign breast disease ( } \mathrm{n}, \\
\text { \%yes) }\end{array}$ & $\begin{array}{l}147 \\
46.7\end{array}$ & $89,25.6^{\star}$ & $37,44.0$ & $25,18.7^{\star}$ & $110,47.6$ & $64,29.9 *$ \\
\hline \multirow[t]{2}{*}{ Hormone therapy (n, \% ever) } & / & / & / & / & $86,38.2$ & $61,29.6$ \\
\hline & & & & & $(n=225)$ & $(n=206)$ \\
\hline
\end{tabular}

When anthropometric characteristics were compared between cases and controls (Table 2), cases had lower BMI, waist circumference, and WHtR. In analyses of anthropometric characteristics by menopausal status (Table 2), there were no statistically significant differences between premenopausal cases and controls. In the postmenopausal group, cases were taller, and had lower waist circumference, WHtR, and WHR than controls. Although BMI was not significantly different between premenopausal cases and controls, on average, both were in the obese category. Postmenopausal controls were in the obese category, and postmenopausal cases were borderline between overweight and obese. Considering the average percent body fat estimated from skinfolds, all pre and postmenopausal cases and controls were in the obese category (Table 2). Sitting height and ABSI (not shown in Table 2) were similar between cases and controls with a mean of $0.80(S D=0.005) \mathrm{m}$, and $0.075(\mathrm{SD}=0.006) \mathrm{m}^{11 / 6} \mathrm{~kg}^{-2 / 3}$, respectively. 
Table 2

Anthropometric Characteristics of Study Participants by Case-control Status, and by Menopausal Status

\begin{tabular}{|c|c|c|c|c|c|c|}
\hline \multirow[t]{2}{*}{ Characteristic } & \multirow{2}{*}{$\begin{array}{l}\text { All } \\
\text { Cases } \\
(n=315)\end{array}$} & \multirow{2}{*}{$\begin{array}{l}\text { All } \\
\text { Controls } \\
(n=348)\end{array}$} & \multicolumn{2}{|c|}{ Premenopausal } & \multicolumn{2}{|c|}{ Postmenopausal } \\
\hline & & & $\begin{array}{l}\text { Cases } \\
(n=84)\end{array}$ & $\begin{array}{l}\text { Controls } \\
(n=134)\end{array}$ & $\begin{array}{l}\text { Cases } \\
(n=231)\end{array}$ & $\begin{array}{l}\text { Controls } \\
(n=214)\end{array}$ \\
\hline Weight (kg) & $71.7(14.8)$ & $74.1(17.9)$ & $74.1(16.9)$ & $77.7(20.8)$ & $70.9(13.9)$ & $71.8(15.5)$ \\
\hline Lifetime weight gain $(\mathrm{kg})$ & $\begin{array}{l}19.3(12.9) \\
(n=306)\end{array}$ & $\begin{array}{l}19.4(14.6) \\
(n=335)\end{array}$ & $\begin{array}{l}20.0(14.6) \\
(n=81)\end{array}$ & $\begin{array}{l}19.7(16.3) \\
(n=130)\end{array}$ & $\begin{array}{l}19.0(12.3) \\
(n=225)\end{array}$ & $\begin{array}{l}19.3(13.5) \\
(n=205)\end{array}$ \\
\hline Height (m) & $\begin{array}{l}1.55(0.07) \\
(n=311)\end{array}$ & $\begin{array}{l}1.54(0.08) \\
(n=347)\end{array}$ & $1.57(0.07)$ & $\begin{array}{l}1.57(0.08) \\
(n=133)\end{array}$ & $\begin{array}{l}1.54(0.07)^{\mathrm{d}} \\
(n=227)\end{array}$ & $\begin{array}{l}1.53(0.07)^{d} \\
(n=214)\end{array}$ \\
\hline Body mass index $\left(\mathrm{kg} / \mathrm{m}^{2}\right)$ & $\begin{array}{l}30.0(6.0)^{a} \\
(n=311)\end{array}$ & $\begin{array}{l}31.2(7.4)^{a} \\
(n=347)\end{array}$ & $30.2(6.6)$ & $\begin{array}{l}31.7(8.7) \\
(n=133)\end{array}$ & $\begin{array}{l}29.9(5.7) \\
(n=227)\end{array}$ & $\begin{array}{l}30.8(6.5) \\
(n=214)\end{array}$ \\
\hline Waist circumference $(\mathrm{cm})$ & $\begin{array}{l}89.3(12.7)^{\mathrm{b}} \\
(n=306)\end{array}$ & $\begin{array}{l}91.7(13.7)^{\mathrm{b}} \\
(n=340)\end{array}$ & $88.2(14.0)$ & $\begin{array}{l}90.5(15.3) \\
(n=132)\end{array}$ & $\begin{array}{l}89.7(12.2)^{\mathrm{e}} \\
(n=222)\end{array}$ & $\begin{array}{l}92.5(12.5)^{\mathrm{e}} \\
(n=208)\end{array}$ \\
\hline Waist to height ratio & $\begin{array}{l}0.58(0.08)^{\mathrm{c}} \\
(n=306)\end{array}$ & $\begin{array}{l}0.60(0.09)^{\mathrm{c}} \\
(n=340)\end{array}$ & $0.56(0.09)$ & $\begin{array}{l}0.58(0.10) \\
(n=132)\end{array}$ & $\begin{array}{l}0.58(0.08)^{f} \\
(n=222)\end{array}$ & $\begin{array}{l}0.61(0.08)^{f} \\
(n=208)\end{array}$ \\
\hline Waist to hip ratio & $\begin{array}{l}0.85(0.07) \\
(n=306)\end{array}$ & $\begin{array}{l}0.86(0.08) \\
(n=340)\end{array}$ & $0.83(0.08)$ & $\begin{array}{l}0.83(0.07) \\
(n=132)\end{array}$ & $\begin{array}{l}0.86(0.07)^{g} \\
(n=222)\end{array}$ & $\begin{array}{l}0.87(0.08)^{g} \\
(n=208)\end{array}$ \\
\hline Body fat (\%) & $\begin{array}{l}40.8(8.2) \\
(n=299)\end{array}$ & $\begin{array}{l}41.8(10.7) \\
(n=331)\end{array}$ & $\begin{array}{l}41.7(8.2) \\
(n=83)\end{array}$ & $\begin{array}{l}42.3(10.6) \\
(n=129)\end{array}$ & $\begin{array}{l}40.5(8.1) \\
(n=216)\end{array}$ & $\begin{array}{l}41.5(10.7) \\
(n=202)\end{array}$ \\
\hline Lean body mass (kg) & $\begin{array}{l}41.7(8.0) \\
(n=299)\end{array}$ & $\begin{array}{l}42.0(8.6) \\
(n=331)\end{array}$ & $\begin{array}{l}42.2(7.2) \\
(n=83)\end{array}$ & $\begin{array}{l}43.2(8.6) \\
(n=129)\end{array}$ & $\begin{array}{l}41.6(8.3) \\
(n=216)\end{array}$ & $\begin{array}{l}41.3(8.6) \\
(n=202)\end{array}$ \\
\hline
\end{tabular}

Correlation analysis among the anthropometric variables for premenopausal and postmenopausal women are shown in Table 3 (only statistically significant correlations). Most anthropometric variables in pre and postmenopausal cases and controls were significantly correlated. The strongest correlations $(\geq 0.8)$ between anthropometric variables not sharing one or more measures are in bold. In premenopausal cases the strongest correlation was for BMI and lifetime weight gain (0.80). In postmenopausal cases, the strongest correlation was for $\mathrm{BMI}$ and waist circumference $(0.80)$. In premenopausal controls, the strongest correlations were observed for $\mathrm{BMI}$ and waist circumference (0.93), BMI and lifetime weight gain 0.84), and waist circumference and lifetime weight gain (0.83). For postmenopausal controls, none of the correlations were at or above 0.8 . 
Table 3

Significant Spearman Correlations among Anthropometric Variables by Case-control and Menopausal Status

\begin{tabular}{|c|c|c|c|c|c|c|c|c|c|c|}
\hline $\begin{array}{l}\text { Anthropometric } \\
\text { Variable }\end{array}$ & $\begin{array}{l}\text { LWG } \\
(\mathrm{kg})\end{array}$ & $\begin{array}{l}\text { BMI } \\
\left(\mathrm{kg} / \mathrm{m}^{2}\right)\end{array}$ & $\begin{array}{l}\text { Waist-C } \\
\text { (cm) }\end{array}$ & WHtR & WHR & $\begin{array}{l}\text { LWG } \\
(\mathbf{k g})\end{array}$ & $\begin{array}{l}\text { BMI } \\
\left(\mathrm{kg} / \mathrm{m}^{2}\right)\end{array}$ & $\begin{array}{l}\text { Waist-C } \\
\text { (cm) }\end{array}$ & WHtR & WHR \\
\hline & \multicolumn{5}{|c|}{ Premenopausal Cases } & \multicolumn{5}{|c|}{ Postmenopausal Cases } \\
\hline Lifetime WG (kg) & \multicolumn{5}{|l|}{ / } & \multicolumn{5}{|l|}{ / } \\
\hline $\mathrm{BMI}\left(\mathrm{kg} / \mathrm{m}^{2}\right)$ & $0.80 *$ & \multicolumn{4}{|l|}{ / } & $0.79 *$ & \multicolumn{4}{|l|}{ / } \\
\hline Waist C (cm) & $0.70 *$ & $0.78 *$ & \multicolumn{3}{|l|}{ / } & $0.68^{*}$ & $0.80 *$ & \multicolumn{3}{|l|}{ / } \\
\hline WHtR & $0.67 *$ & $0.84^{\star}$ & $0.94 *$ & \multicolumn{2}{|l|}{ / } & $0.61 *$ & $0.84 *$ & $0.94^{*}$ & \multicolumn{2}{|l|}{ / } \\
\hline WHR & $0.30 *$ & $0.28 *$ & $0.68 *$ & $0.66 *$ & / & $0.23^{*}$ & $0.30 *$ & $0.59 *$ & $0.61 *$ & / \\
\hline Body fat (\%) & $0.57 *$ & $0.64^{\star}$ & $0.50 *$ & $0.49 *$ & & $0.30 *$ & $0.36^{\star}$ & $0.27 *$ & $0.29 *$ & \\
\hline \multirow{2}{*}{$\begin{array}{l}\text { Lean body mass } \\
(\mathrm{kg})\end{array}$} & $0.53^{\star}$ & $0.59 *$ & $0.60 *$ & $0.50 *$ & $0.25^{\star}$ & $0.60 *$ & $0.61 *$ & $0.60 *$ & $0.48 *$ & $0.22^{*}$ \\
\hline & \multicolumn{5}{|c|}{ Premenopausal Controls } & \multicolumn{5}{|c|}{ Postmenopausal Controls } \\
\hline Lifetime WG (kg) & \multicolumn{5}{|l|}{ / } & \multicolumn{5}{|l|}{ / } \\
\hline $\mathrm{BMI}\left(\mathrm{kg} / \mathrm{m}^{2}\right)$ & $0.84 *$ & \multicolumn{4}{|l|}{ / } & $0.74^{\star}$ & \multicolumn{4}{|l|}{ / } \\
\hline Waist C (cm) & $0.83^{*}$ & $0.93^{*}$ & \multicolumn{3}{|l|}{ I } & $0.64 *$ & $0.72^{\star}$ & \multicolumn{3}{|l|}{ / } \\
\hline WHtR & $0.78^{*}$ & $0.91 *$ & $0.96 *$ & \multicolumn{2}{|l|}{ / } & $0.56^{\star}$ & $0.78^{\star}$ & $0.93^{\star}$ & \multicolumn{2}{|l|}{ / } \\
\hline WHR & $0.38^{*}$ & $0.39 *$ & $0.59 *$ & $0.65^{\star}$ & / & & & $0.55^{\star}$ & $0.56^{\star}$ & / \\
\hline Body fat (\%) & $0.74^{\star}$ & $0.75^{\star}$ & $0.70 *$ & $0.67 *$ & $0.29 *$ & $0.53^{*}$ & $0.50 *$ & $0.36^{\star}$ & $0.32^{\star}$ & \\
\hline $\begin{array}{l}\text { Lean body mass } \\
(\mathrm{kg})\end{array}$ & $0.39 *$ & $0.54^{\star}$ & $0.54^{\star}$ & \multicolumn{2}{|l|}{$0.41^{\star}$} & $0.37 \star$ & $0.49 *$ & $0.55^{\star}$ & $0.47^{\star}$ & $0.25^{\star}$ \\
\hline \multicolumn{11}{|c|}{${ }^{*} p<0.05, \mathrm{LWG}=$ lifetime weight gain, $\mathrm{BMI}=$ body mass index, Waist $\mathrm{C}=$ waist circumference, $\mathrm{WHtR}=$ waist to height ratio, } \\
\hline
\end{tabular}

The odds of breast cancer associated with each anthropometric variable by menopausal status with significant trend are shown in Table 4. There was a significant trend toward lower odds of premenopausal breast cancer for those in the highest compared to the lowest tertile of BMI ( $P$ for trend $=0.03$ ) and WHtR ( $P$ for trend $=0.05)$. The odds of postmenopausal breast cancer were significantly lower among those in the highest tertile of waist circumference ( $P$ for trend=0.05), WHtR ( $P$ for trend= 0.01), and WHR (P for trend= 0.05); and significantly higher among those in the highest tertile of height ( $P$ for trend = 0.05). The risk of premenopausal or postmenopausal breast cancer was not significantly associated with lifetime weight gain, sitting height, sitting height to height ratio, $\mathrm{ABSI}$, sum of skinfolds or percent body fat and lean body mass estimated from skinfold thicknesses. 
Anthropometric Characteristics and Risk of Breast Cancer by Menopausal Status

\begin{tabular}{|c|c|c|c|c|c|}
\hline Characteristics* & Cases & Controls & Crude OR (Cl) & Adjusted OR** (Cl) & $P$ for trend** \\
\hline \multicolumn{6}{|c|}{ Body mass index $\left(B M I=k g / m^{2}\right)$} \\
\hline \multicolumn{6}{|l|}{ Premenopausal } \\
\hline$<25$ & 21 & 31 & 1.00 & 1.00 & 0.03 \\
\hline $25-29.9$ & 28 & 37 & $1.12(0.53-2.34)$ & $1.01(0.41-2.49)$ & \\
\hline$\geq 30$ & 35 & 65 & $0.80(0.40-1.58)$ & $0.45(0.19-1.07)$ & \\
\hline \multicolumn{6}{|l|}{ Postmenopausal } \\
\hline$<25$ & 45 & 36 & 1.00 & 1.00 & 0.14 \\
\hline $25-29.9$ & 81 & 60 & $1.08(0.62-1.87)$ & $1.08(0.60-1.94)$ & \\
\hline$\geq 30$ & 101 & 118 & $0.69(0.41-1.14)$ & $0.67(0.39-1.16)$ & \\
\hline \multicolumn{6}{|c|}{ Waist circumference (cm) } \\
\hline \multicolumn{6}{|l|}{ Premenopausal } \\
\hline$<83.8$ & 32 & 43 & 1.00 & 1.00 & 0.07 \\
\hline $83.8-95.3$ & 29 & 46 & $0.85(0.44-1.63)$ & $0.75(0.34-1.68)$ & \\
\hline$>95.3$ & 23 & 43 & $0.72(0.36-1.42)$ & $0.48(0.20-1.14)$ & \\
\hline \multicolumn{6}{|l|}{ Postmenopausal } \\
\hline$<86.4$ & 94 & 70 & 1.00 & 1.00 & 0.05 \\
\hline $86.4-97.2$ & 75 & 70 & $0.80(0.51-1.25)$ & $0.84(0.52-1.35)$ & \\
\hline$>97.2$ & 53 & 68 & $0.58(0.36-0.93)$ & $0.61(0.37-1.02)$ & \\
\hline \multicolumn{6}{|c|}{ Waist to height ratio (WHtR) } \\
\hline \multicolumn{6}{|l|}{ Premenopausal } \\
\hline$<0.53$ & 30 & 44 & 1.00 & 1.00 & 0.05 \\
\hline $0.53-0.62$ & 35 & 44 & $1.17(0.61-2.22)$ & $0.81(0.36-1.82)$ & \\
\hline$>0.62$ & 19 & 44 & $0.63(0.31-1.29)$ & $0.36(0.15-0.89)$ & \\
\hline \multicolumn{6}{|l|}{ Postmenopausal } \\
\hline$<0.57$ & 100 & 69 & 1.00 & 1.00 & 0.01 \\
\hline $0.57-0.64$ & 65 & 71 & $0.63(0.40-0.99)$ & $0.68(0.42-1.11)$ & \\
\hline$>0.64$ & 57 & 68 & $0.58(0.36-0.92)$ & $0.59(0.36-0.97)$ & \\
\hline
\end{tabular}

*Tertiles among controls by menopausal status were used as cutoffs to define the categories for all anthropometric variables, except BMI. ** Odds ratios and $95 \%$ confidence intervals adjusted for age, education, age at menarche, parity, age at menopause (post-only), previous benign breast disease, and family history of breast cancer. 


\begin{tabular}{|c|c|c|c|c|c|}
\hline Characteristics* & Cases & Controls & Crude OR (Cl) & Adjusted OR** (Cl) & $P$ for trend** \\
\hline \multicolumn{6}{|c|}{ Waist to hip ratio (WHR) } \\
\hline \multicolumn{6}{|l|}{ Premenopausal } \\
\hline$<0.80$ & 27 & 44 & 1.00 & 1.00 & 0.50 \\
\hline $0.80-0.87$ & 31 & 44 & $1.15(0.59-2.23)$ & $1.11(0.50-2.46)$ & \\
\hline$>0.87$ & 26 & 44 & $0.96(0.49-1.90)$ & $0.76(0.34-1.74)$ & \\
\hline \multicolumn{6}{|l|}{ Postmenopausal } \\
\hline$<0.84$ & 86 & 69 & 1.00 & 1.00 & 0.04 \\
\hline $0.84-0.90$ & 80 & 70 & $0.92(0.58-1.44)$ & $1.03(0.64-1.68)$ & \\
\hline$>0.90$ & 56 & 69 & $0.65(0.41-1.05)$ & $0.71(0.43-1.19)$ & \\
\hline \multicolumn{6}{|l|}{ Height (m) } \\
\hline \multicolumn{6}{|l|}{ Premenopausal } \\
\hline$<1.55$ & 36 & 53 & 1.00 & 1.00 & 0.81 \\
\hline $1.55-1.60$ & 26 & 44 & $0.87(0.46-1.66)$ & $1.17(0.55-2.45)$ & \\
\hline$>1.60$ & 22 & 36 & $0.90(0.46-1.77)$ & $0.95(0.43-2.12)$ & \\
\hline \multicolumn{6}{|l|}{ Postmenopausal } \\
\hline$<1.50$ & 64 & 73 & 1.00 & 1.00 & 0.04 \\
\hline $1.50-1.56$ & 83 & 76 & $1.25(0.79-1.97)$ & $1.18(0.72-1.92)$ & \\
\hline$>1.56$ & 80 & 65 & $1.40(0.88-2.24)$ & $1.42(0.85-2.37)$ & \\
\hline
\end{tabular}

Significant association of anthropometric measures with breast cancer in strata of HT are presented in Table 5. Lower odds of breast cancer were observed for postmenopausal participants taking HR who were in the highest tertile for waist circumference ( $P$ for trend= 0.01), WHtR ( $P$ for trend= 0.01), WHR ( $P$ for trend= 0.03), and ABSI (P for trend=0.02). For postmenopausal participants not taking $\mathrm{HT}$, a higher risk of breast cancer was observed for those in the highest tertile of height ( $P$ for trend=0.04). 
Table 5

Anthropometric Characteristics and Risk of Breast Cancer among Post-menopausal Women, Stratified by Hormone Therapy (HT)

\begin{tabular}{|c|c|c|c|c|c|c|c|c|c|c|}
\hline Characteristics* & Cases & Controls & $\begin{array}{l}\text { Crude } \\
\text { OR }\end{array}$ & $\begin{array}{l}\text { Adjusted } \\
\text { OR** } \\
\text { (Cl) }\end{array}$ & $\begin{array}{l}P \text { for } \\
\text { trend** }\end{array}$ & Cases & Controls & $\begin{array}{l}\text { Crude } \\
\text { OR } \\
\text { (Cl) }\end{array}$ & $\begin{array}{l}\text { Adjusted } \\
\text { OR** } \\
\text { (Cl) }\end{array}$ & $\begin{array}{l}P \text { for } \\
\text { trend** }\end{array}$ \\
\hline Yes HT & & & & & & No HT & & & & \\
\hline \multicolumn{11}{|c|}{ Waist circumference $(\mathrm{cm})$} \\
\hline$<86.4$ & 39 & 16 & 1.00 & 1.00 & 0.01 & 51 & 53 & 1.00 & 1.00 & 0.95 \\
\hline \multirow[t]{2}{*}{$86.4-97.2$} & 30 & 22 & $\begin{array}{l}0.56 \\
(0.25- \\
1.25)\end{array}$ & $\begin{array}{l}0.65 \\
(0.27- \\
1.54)\end{array}$ & & 44 & 45 & $\begin{array}{l}1.02 \\
(0.58- \\
1.79)\end{array}$ & $\begin{array}{l}1.06 \\
(0.58- \\
1.96)\end{array}$ & \\
\hline & & & $\begin{array}{l}0.27 \\
(0.11- \\
0.67)\end{array}$ & $\begin{array}{l}0.32 \\
(0.12- \\
0.84)\end{array}$ & & & & $\begin{array}{l}0.92 \\
(0.51- \\
1.64)\end{array}$ & $\begin{array}{l}0.90 \\
(0.48- \\
1.72)\end{array}$ & \\
\hline
\end{tabular}

$>97.2$

$14 \quad 21$

$38 \quad 43$

\section{Waist to height ratio (WHtR)}

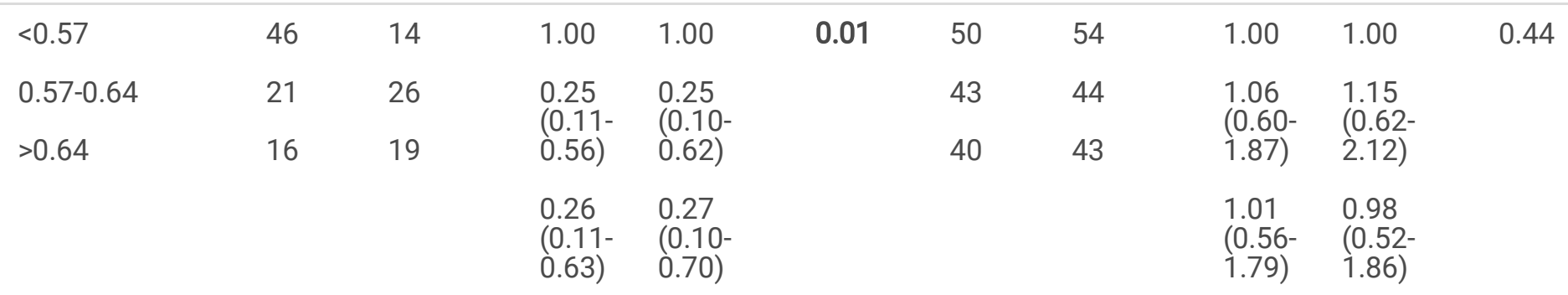

Waist to hip ratio (WHR)

\begin{tabular}{|c|c|c|c|c|c|c|c|c|c|c|}
\hline$<0.84$ & 39 & 16 & 1.00 & 1.00 & 0.03 & 43 & 53 & 1.00 & 1.00 & 0.59 \\
\hline $0.84-0.90$ & 29 & 17 & \multirow{2}{*}{$\begin{array}{l}0.70 \\
(0.30- \\
1.61)\end{array}$} & \multirow{2}{*}{$\begin{array}{l}0.68 \\
(0.28- \\
1.67)\end{array}$} & & 50 & 49 & \multirow{2}{*}{$\begin{array}{l}1.26 \\
(0.72- \\
2.21)\end{array}$} & \multirow{2}{*}{$\begin{array}{l}1.41 \\
(0.77- \\
2.60)\end{array}$} & \\
\hline$>0.90$ & 15 & 26 & & & & 40 & 39 & & & \\
\hline & & & $\begin{array}{l}0.24 \\
(0.10- \\
0.56)\end{array}$ & $\begin{array}{l}0.25 \\
(0.10- \\
0.65)\end{array}$ & & & & $\begin{array}{l}1.26 \\
(0.70- \\
2.30)\end{array}$ & $\begin{array}{l}1.35 \\
(0.71- \\
2.59)\end{array}$ & \\
\hline
\end{tabular}

A Body Shape Index (ABSI) $\left(\mathrm{m}^{11 / 6} \mathrm{~kg}^{-2 / 3}\right)$

$\begin{array}{lllllllllll}<0.074 & 33 & 14 & 1.00 & 1.00 & 0.02 & 41 & 55 & 1.00 & 1.00 & 0.44 \\ 0.074-0.079 & 27 & 18 & 0.64 & 0.52 & & 52 & 51 & 1.37 & 1.25 \\ >0.079 & 23 & 27 & (0.27- & (0.20- & & & & (0.78- & (0.68- \\ & & & 1.51) & 1.36) & & 40 & 35 & 2.39) & 2.30) \\ & & & 0.36 & 0.32 & & & & 1.53 & 1.61 \\ & & & (0.16- & (0.12- & & & & (0.84- & (0.84- \\ & & & & & & & & & 2.82) & 3.08)\end{array}$

Height (m)

*Tertiles among controls by menopausal status were used as cutoffs to define the categories for all anthropometric variables, except BMI. ** Odds ratios and 95\% confidence intervals adjusted for age, education, age at menarche, parity, age at menopause, previous benign breast disease, and family history of breast cancer. 


\begin{tabular}{|c|c|c|c|c|c|c|c|c|c|c|}
\hline Characteristics* & Cases & Controls & $\begin{array}{l}\text { Crude } \\
\text { OR }\end{array}$ & $\begin{array}{l}\text { Adjusted } \\
\text { OR** } \\
\text { (Cl) }\end{array}$ & $\begin{array}{l}P \text { for } \\
\text { trend** }\end{array}$ & Cases & Controls & $\begin{array}{l}\text { Crude } \\
\text { OR } \\
\text { (Cl) }\end{array}$ & $\begin{array}{l}\text { Adjusted } \\
\text { OR** } \\
\text { (Cl) }\end{array}$ & $\begin{array}{l}P \text { for } \\
\text { trend** }\end{array}$ \\
\hline$<1.50$ & 27 & 18 & 1.00 & 1.00 & 0.83 & 36 & 52 & 1.00 & 1.00 & 0.04 \\
\hline \multirow[t]{2}{*}{$1.50-1.56$} & 31 & 23 & $\begin{array}{l}0.90 \\
(0.40- \\
2.01)\end{array}$ & $\begin{array}{l}0.99 \\
(0.41- \\
2.37)\end{array}$ & & 48 & 52 & $\begin{array}{l}1.33 \\
(0.75- \\
2.38)\end{array}$ & $\begin{array}{l}1.17 \\
(0.63- \\
2.18)\end{array}$ & \\
\hline & & & $\begin{array}{l}0.93 \\
(0.41- \\
2.14)\end{array}$ & $\begin{array}{l}0.92 \\
(0.38- \\
2.25)\end{array}$ & & & & $\begin{array}{l}1.80 \\
(0.99- \\
3.25)\end{array}$ & $\begin{array}{l}1.79 \\
(0.92- \\
3.50)\end{array}$ & \\
\hline$>1.56$ & 28 & 20 & & & & 51 & 41 & & & \\
\hline
\end{tabular}

\section{Discussion}

To our knowledge, this case-control study is the first population-based study regarding breast cancer risk in relation to anthropometric characteristics among women in PR. Women in the study, both pre-and postmenopausal and both cases and controls, were, on average, close to obese, with an average BMI similar to but slightly lower body weight, height, and waist circumference of Hispanic women in the continental USA [33]. When compared with white and black women, our participants had on average higher BMI, and slightly lower waist circumference, height, and weight. No significant differences between cases and controls were observed for mean lifetime weight gain and estimated percent body fat; 19 to $20 \mathrm{~kg}$ of lifetime weight gain, and $40 \%$ of estimated body fat are indicative of excess weight gain and body fat. We observed WHtR above 0.50 and waist circumference above $88 \mathrm{~cm}$ among pre- and postmenopausal cases and controls; both strongly associated with central and general body fat accumulation [34-36]. WHR higher than 0.85 place postmenopausal participants at risk of cardiovascular and metabolic disease [34].

The anthropometric measures correlated strongly and directly with each other; those with high BMI also tended to have high waist circumference and/or WHtR. The combination of high BMI, high waist circumference, and high WHtR has been proposed to improve risk detection for cardiovascular and metabolic disease. For example, Luz, Barbosa and d'Orsi [37] reported that the combination of high BMI and waist circumference or BMI and WHtR provide a strong indication of risk for hypertension. Also, Zhang et al. [38] reported that the combination of high BMI and waist circumference added value in explaining risk of hypertension among Chinese women. In a meta-analysis, Seo et al. [39], reported that BMl equal or higher than $30 \mathrm{~kg} / \mathrm{m}^{2}$ combined with a waist circumference equal or higher than $88 \mathrm{~cm}$ places women at a very high risk of obesity and type 2 diabetes, hypertension, and cardiovascular disease compared with women within normal BMI and waist circumference at or less than $88 \mathrm{~cm}$. The authors also showed differences by race/ethnicity in that only among Hispanic WC equal or higher than $88 \mathrm{~cm}$, not BMI equal or higher than 30, predicted development of hypertension among Hispanic/Latinos. Although race/ethnicity could also play a role in how anthropometric characteristics relate to breast cancer risk, published studies including women from Hispanic background could not be found.

$\mathrm{BMI}$, waist circumference, and WHtR were anthropometric characteristics of concern in our study because of their association with breast cancer as well as cardiovascular and metabolic disease. However, high BMI and high WHtR in premenopausal participants, and high waist circumference, high WHtR and high WHR in postmenopausal participants were associated with lower odds of breast cancer in our study. These results are not consistent with previous reports showing high BMI as a risk factor for postmenopausal breast cancer but are consistent with the findings of others of reduced risk associated with premenopausal breast cancer [13]. Our results also differ from Gravena et al. [40] who reported a higher risk of breast cancer only among postmenopausal women in Brazil with a BMI equal or higher than $30 \mathrm{~kg} / \mathrm{m}^{2}$, and no association for 
premenopausal women. Chen et al. [41], in their dose-response meta-analysis of 31 cohort studies, reported a direct association between BMI and postmenopausal breast cancer risk, but no association for premenopausal breast cancer. There is evidence that the association of BMI with postmenopausal risk differs by HT use [42], suggesting a positive association with breast cancer risk only among HT users. Although BMI was not significantly associated with breast cancer risk regardless of HT use in our study, we found an inverse association of waist circumference, WHR, and WHtR with breast cancer risk among those taking HT. There might be differences in the hormonal metabolism and etiology related to breast cancer for Puerto Rican women that should be further. To our knowledge, there are no such studies among Hispanic women.

Consistent with previous studies [13,43-44], we found a significant trend of higher odds of breast cancer associated with height, but only among postmenopausal participants. Mellemkjar et al. [45] also reported higher risk of postmenopausal breast cancer in taller women, with both components of height (leg length and sitting height) equally influencing the risk. We also found that sitting height increased the odds of postmenopausal breast cancer. Although height and sitting height have been related to growth hormone exposure and childhood nutrition [45-46], the specific biological mechanisms explaining adult height or sitting height attainment on breast cancer risk are yet to be determined. Identifying a common regulatory pathway for cellular growth that potentially affects adult height attainment and cancer development [47] needs to be addressed in future research.

There is a proposed biological mechanism that links excess body weight, especially abdominal adiposity to metabolic and hormonal disturbances associated with breast cancer risk, such as insulin resistance, inflammation, and exposure to estrogen production via aromatization of androgens [15, 48-49]. However, we found that higher values for both BMI and the indirect measure of abdominal adiposity were associated with decreased breast cancer risk in both pre- and postmenopausal women in Puerto Rico. In addition, different from what has been reported elsewhere is our observation of an inverse association of higher waist circumference with risk among postmenopausal women on HT.

Also different from previous publications [13,50], excess lifetime weight gain and percent fat were not associated with breast cancer risk. Keum, et al. [48] found an increased risk in postmenopausal breast cancer for each $5 \mathrm{~kg}$ increase in adult weight gain up to $35 \mathrm{~kg}$, only among women not taking HT. Our results differed in that lifetime weight gain and excess adiposity were not associated with pre- and postmenopausal breast cancer risk irrespective of HT. There are important sources of error in body fat and lean body mass estimated from skinfold thicknesses, including the use of equations with unknown validity in specific study populations like ours, and the lack of information regarding body distribution of these tissues. Therefore, conclusions from our results regarding the association between percent body fat, lean body mass, and risk of breast cancer in pre and postmenopausal Hispanic women in PR are limited at this point.

This study had some important strengths. This is the first population-based case control study of breast cancer risk in PR. The focus on a Hispanic population is significant. In addition, the extensive measurement of anthropometry is a strength. Because the prevalence and incidence rates of breast cancer are different in PR than in the USA [26], understanding breast cancer risk associated with anthropometric measures in women with Hispanic background in PR is clearly needed. The study was limited by the sample size, affecting the study power and our ability to do analyses of sub-groups, particularly the analyses within strata of HT. As with any case-control study, there are issues of misclassification and confounding. However, the measures were made in a standardized fashion, with interviewers blinded to the participants' case control status. We adjusted for potential confounders to the extent possible. There may be some residual confounding. Finally, the anthropometric measures were made for the cases after breast cancer diagnosis. There may be some changes in weight status with disease development. Most of the study participants had early-stage disease and were less likely to have experienced weight loss as a result of cancer.

In conclusion, in this population-based case-control study of Hispanic women in Puerto Rico, we found associations of anthropometric measurements with breast cancer risk. Premenopausal women had lower odds of breast cancer associated with BMI at $25 \mathrm{~kg} / \mathrm{m}^{2}$ or higher, and a WHtR of 0.53 or higher. Postmenopausal women had lower odds of breast cancer associated with a waist circumference of $86.4 \mathrm{~cm}$ or higher, WHtR of 0.57 or higher, WHR of 0.84 or higher, and height of 150 
$\mathrm{cm}$ or taller. Understanding potential differences in risk factors for Hispanic women is critical, particularly those related to metabolic disturbances. Future studies should include direct measures of body fat and lean mass distribution to further understand the predictive value of anthropometric measures in the assessment of breast cancer risk among Hispanic women.

\section{Declarations}

\section{Acknowledgements}

The Atabey Epidemiology of Breast Cancer in Puerto Rico Study was supported by CDMRP Grant W81XWH-07-1-0329 BCRP HBCU/MI Partnership Training Award. The views, opinions or endorsements are solely the responsibility of the authors and do not necessarily reflect the official views of supporting agencies.

The research team acknowledges The Consortium of Clinical and Translational Research of Puerto Rico (NIMHD and NIAID award number 2U54MD007587), the Puerto Rico Central Cancer Registry, many collaborating physicians and their affiliated institutions, the interviewers, the community outreach worker, and the graduate students from the UPR Graduate School of Public Health who contributed to this research.

Authors' contributions: All authors contributed to the study conception and design, including material preparation, data collection and analyses. The first draft of the manuscript was written by Farah A. Ramírez-Marrero and all authors commented on previous versions of the manuscript. All authors read and approved the final manuscript.

Funding: Congressionally Directed Medical Research Programs (CDMRP) Grant W81XWH-07-1-0329 BCRP, Historically Black Colleges and Universities/Minority Institutions (HBCU/MI) Partnership Training Award. National Institute on Minority Health and Health Disparities (NIMHD) and National Institute of Allergy and Infectious Diseases (NIAID) award number 2U54MD007587.

Conflicts of interest/Competing interests: The authors have no conflict of interest to declare that are relevant to the content of this article.

Availability of data and material: The datasets generated and analyzed during the current study are available from the corresponding author upon reasonable request.

Code availability: not applicable

Ethics approval: The study was approved by the Institutional Review Board of the University of Puerto Rico Medical Sciences Campus.

Consent to participate: Informed consent was obtained from all individual participants in the study.

Consent for publication: Participants signed informed consent regarding publication of study data without direct or indirect identifiers.

\section{References}

1. Flegal KM, Kit BK, Orpana H, Graubard BI (2013) Association of all-cause mortality with overweight and obesity using standard body mass index categories: a systematic review and meta-analysis. JAMA 309:71-82.

https://doi.org/10.1001/jama.2012.113905

2. Mechanick JI, Hurley DL, Garvey WT (2017) Adiposity-based chronic disease as a new diagnostic term: The American Association of Clinical Endocrinologists and American College of Endocrinology position statement. Endocr Pract 23(3):1-7. https://doi.org/10.4158/EP161688.PS 
3. Manolopoulos KN, Karpe F, Frayn KN (2010) Gluteofemoral body fat as a determinant of metabolic health. Int J Obes (Lond) 34:949-959. https://doi.org/10.1038/ijo.2009.286

4. Lorenzini A (2014) How much should we weight for a long and healthy life span? The need to reconcile caloric restriction versus longevity with body mass index versus mortality data. Front Endocrinol 5(121):1-8.

https://doi.org/10.3389/fendo.2014.00121

5. Deurenberg-Yap M, Schmidt G, van Staveren WA, Deurengerg P (2000) The paradox of low body mass index and high body fat percentage among Chinesse, Malays and Indians in Singapore. Int J Obes Relat Metab Disord 24:1011-7. https://doi.org/10.1038/sj.ijo.0801353

6. World Health Organization Expert Consultation (2004) Appropriate body-mass index for Asian populations and its implications for policy and intervention strategies. Lancet 363:157-63. https://doi.org/10.1016/S0140-6736(03)15268-3

7. Rush EC, Goedecke JH, Jennings C, Micklesfield L, Dugas L, Lambert EV, Plank LD (2007) BMI, fat and muscle differences in urban women of five ethnicities from two countries. Int J Obes 31:1232-1239. https://doi.org/10.1038/sj.ijo.0803576

8. Herrera VM, Casas JP, Miranda JJ, Perel P, Pichardo R, González A, Sanchez JR, Ferreccio C, Aguilera X, Silva E, Oróstegui M, Gómez LF, Chirinos JA, Medina-Lezama J, Pérez CM, Suárez E, Ortiz AP, Rosero L, Schapochnik N, Ortiz Z, Ferrante D, Diaz M, Bautista LE (2009) Interethnic differences in the accuracy of anthropometric indicators of obesity in screening for high risk of coronary heart disease. Int J Obes (Lon) 33(5): 568-576. https://doi.org/10.1038/ijo.2009.35

9. IARC- International Agency for Research on Cancer (2002) Weight control and physical activity. IARC Handbooks of Cancer Prevention, Volume 6. Lyon, France; IARC Press.

10. WCRF/AICR - World Cancer Research Fund / American Institute for Cancer Research (2007) Food, Nutrition, Physical Activity, and the Prevention of Cancer: a Global Perspective. Washington, DC: AICR.

11. Han D, Nie J, Bonner MR, McCann SE, Muti P, Trevisan M, Ramirez-Marrero FA, Vito D, Freudenheim JL (2006) Lifetime adult weight gain, central adiposity, and the risk of pre and postmenopausal breast cancer in the Western New York exposures and breast cancer study. Int J Cancer 119:2931-2937. https://doi.org/10.1002/ijc.22236

12. Chen X, Lu W, Zheng W, Gu K, Chen Z, Zheng Y, Shu XO (2010) Obesity and weight change in relation to breast cancer survival. Breast Cancer Res Treat 122:823-833. https://doi.org/10.1007/s10549-009-0708-3

13. WCRF/AICR (2018) Body fatness and weight gain and the risk of cancer. London, World Cancer Research Fund International.

14. The Premenopausal Breast Cancer Collaboration Group (2018) Association of body mass index and age with subsequent breast cancer risk in premenopausal women. JAMA Oncology 4(11):e181771. https://doi.org/10.1001/jamaoncol.2018.1771

15. Goddinho-Mota JCM, Martins KA, Vaz-Goncalves L, Mota JF, Soares LR, Freitas-Junior R (2018) Visceral adiposity increases the risk of breast cancer: a case-control study. Nutr Hosp 35:576-581. https://doi.org/10.20960/nh.1441

16. Wenten M, Gilliand FD, Baumgartner K, Samet JM (2002) Associations of weight, weight change, and body mass with breast cancer risk in Hispanic and non-Hispanic white women. Ann Epidemiol 12:435-444. https://doi.org/10.1016/s10472797(01)00293-9

17. Slattery ML, Sweeney C, Edwards S, Herrick J, Baumgartner L, Wolf R, Murtaugh M, Baumgartner R, Giuliano A, Byers T (2007) Body size, weight change, fat distribution and breast cancer risk in Hispanic and non-Hispanic white women. Breast Cancer Res Treat 102:85-101. https://doi.org/10.1017/s10549-006-9292-y

18. Macciò A, Madeddu C (2011) Obesity, Inflammation, and Postmenopausal Breast 
Cancer: Therapeutic Implications. The Scientific World Journal 11:2020-2036. https://doi.org/10.1100/2011/806787

19. Männistö S, Pietinen P, Pyy M, Palmgren J, Eskelinen M, Uusitupa M (1996) Body-size indicators and risk of breast cancer according to menopause and estrogen-receptor status. In J Cancer 68:8-13. https://doi.org/10.1002/(SICI)1097-

0215(19960927)68:1<8::AID-IJC2>3.0C0;2-V

20. Bandera, EV, Chandran U, Zirpoli G, Gong Z, McCann SE, Hong C, Ciupak G, Paulish K, Ambrosone CB (2013) Body fatness and breast cancer risk in women of African ancestry. BMC Cancer 13:475. https://doi.org/10.1186/1471-2407-13-475

21. Harvie, M, Hopper L, Howell AH (2003) Central obesity and breast cancer risk: a systematic review. Obes Rev 4(3):157173. https://doi.org/ 10.1046/j.1467-789x.2003.00108.x

22. Slattery ML, Baumgartner KB, Byers T, Guiliano A, Sweeney C, Herrick J, Curtin K, Murtaugh M, Wolff R (2005) Genetic, anthropometric, and lifestyle factors associated with IGF-1 and IGFBP-3 levels in Hispanic and non-Hispanic white women. Cancer Causes Control 16(10):1147-1157. https://doi.org/10.1007/s10552-005-0318-2

23. Connor, AE, Baumgartner RN, Yang D, Slattery ML, Giuliano AR, Risendal BC, Abdel-Maksoud MM (2012) Differences between Hispanic and non-Hispanic white women with breast cancer for clinical characteristics and their correlates. Ann Epidemiol 23(4):227-232. https://doi.org/10.1016/j.annepidem.2012.12.013

24. Katzmarzyk PT, Mire E, Bray GA, Greenway FL, Heymsfield SB, Bouchard C (2013) Anthropometric markers of obesity and mortality in white and African American adults: The Pennington Center Longitudinal Study. Obesity 21(5):1070-1075. https://doi.org/10.1002/oby.20151

25. CDC - Center for Disease Control and Prevention (2015) Invasive Cancer Incidence - Puerto Rico, 2007-2011. MMWR Morb Mort Wkly Rep 64(14):389-393.

26. Torres-Cintron M, Ortiz AP, Perez-Irizarry J, Soto-Salgado M, Figueroa-Vallés NR, De La Torre-Feliciano T, Ortiz-Ortiz KJ, Calo WA, Suárez-Pérez E (2010) Incidence and mortality of the leading cancer types in Puerto Rico: 1987-2004. PR Health Sci J 29:317-329.

27. Rosario-Rosado RV, Nazario CM, Hernández-Santiago J, Schelske-Santos M, Mansilla-Rivera I, Ramírez-Marrero FA, RamosValencia G, Climent C, Nie J, Freudenheim JL (2020) Breast cancer in a Caribbean population in transition: Design and implementation of the Atabey population-based case-control study of women in the San Juan metropolitan area in Puerto Rico. Int J Environm Res Public Health 17:133. https://doi.org/10.3390/ijerph17041333

28. Heyward V, Wagner D (2004). Applied Body Composition Assessment, $2^{\text {nd }}$ Ed. Champaign, IL, Human Kinetics Publishers.

29. Krakauer NY, Krakauer JC (2012) A new body shape index predicts mortality hazard independently of body mass index. PLoS One 7:e39504. https://doi.org/10.1371/journal.pone.0039504

30. Thompson CA, Garcia DO, Wertheim BC, Hingle MD, Bea JW, Zaslavsky O, Caire-Juvera G, Rohan T, Vitolins MZ, Thompson PA, Lewis CE (2016) Body shape, adiposity index, and mortality in postmenopausal women: Findings from the Women's Health Initiative. Obesity 24:1061-1069. https://doi.org/10.1002/oby.21461

31. Dhana K, Kavousi M, Arfan Ikram M, Tiemeier WH, Hofman A, Franco OH (2016). Body shape index in comparison with other anthropometric measures in prediction of total and cause-specific mortality. J. Epidemiol Community Health 70:90-96. https://doi.org/10.1136/jech-2014-205257

32. Jones ME, Schoemaker MJ, Wright L, McFadden E, Griffin J, Thomas D, Hemming J, Wright K, Ashworth A, Swerdlow AJ (2016) Menopausal hormone therapy and breast cancer: what is the true size of the increased risk? Br J Cancer 115:607-615. https://doi.org/10.1038/bjc.2016.231 
33. Fryar CD, Kruszon-Moran D, Gu Q, Ogden CL (2018) Mean body weight, height, waist circumference, and body mass index among adults: United States, 1999-2000 through 2015-2016. Natl Health Stat Report 122:1-16.

34. World Health Organization (2008) Waist circumference and waist-hip ratio: report of a WHO expert consultation, Geneva, 8-11, December.

35. Browning LM, Hsieh SD, Ashwell M (2010) A systematic review of waist-to-height ratio as a screening tool for the prediction of cardiovascular disease and diabetes: 0.5 could be a suitable global boundary value. Nutr Res Rev 23(2):24769. https://doi.org/ 10.1017/S0954422410000144

36. Swanson MG, Batterham AM, Tsakirides C, Rutherford ZH, Hind K (2017) Prediction of whole-body fat percentage and visceral adipose tissue mass from five anthropometric variables. PLoS One 12(5):e0177175.

https://doi.org/10.1371/journal.pone.0177175

37. Luz RH, Barbosa AR, d'Orsi E (2016) Waist circumference, body mass index and waist-height ratio: Are two indices better than one for identifying hypertension risk in older adults? Prev Med 93:76-81. https://doi.org/10.1016/j.ypmed.2016.09.024

38. Zhang M, Zhao Y, Wang G, Zhang H, Ren Y, Wang B, Zhang L, Yang X, Han C, Pang C, Yin L, Zhao J, Hu D (2016) Body mass index and waist circumference combined predicts obesity-related hypertension better than either alone in a rural Chinese population. Sci Rep 6:31935. https://doi.org/10.1038/srep31935

39. Seo DC, Choe S, Torabi MR (2017) Is waist circumference $\geq 102 / 88 \mathrm{~cm}$ better than body mass index $\geq 30$ to predict hypertension and diabetes development regardless of gender, age group, and race/ethnicity? Meta-analysis. Prev Med 97:100108. https://doi.org/10.1016/j.ypmed.2017.01.012

40. Gravena AAF, Romeiro-Lopes TC, Demitto MO, Borghesan DHP, Dell'Angolo CM, Brischiliari SCR, Carvalho MDB, Pelloso SM (2018) The obesity and the risk of breast cancer among pre and postmenopausal women. Asian Pac J Cancer Prev 19:24292436. https://doi.org/10.2234/APJCP.2018.19.9.2429

41. Chen Y, Liu L, Zhou Q, Imam MU, Cai J, Wang Y, Qi M, Sun P, Ping Z, Fu X (2017) Body mass index and different effects on premenopausal and postmenopausal breast cancer risk: a dose-response meta-analysis with 3,318,796 subjects from 31 cohort studies. BMC Public Health 17:936 (pages 1-11). https://doi.org/10.1186/s12889-017-4953-9

42. Ahn J, Schatzkin A, Lacey JV, Albanes D, Ballard-Barbash R, Adams KF, Kipnis V, Mouw T, Hollenbeck AR, Leitzmann MF (2007) Adiposity, adult weight change, and postmenopausal breast cancer risk. Arch Intern Med 167(19):20912102. https://doi.org/10.1001/archinte.167.19.2091

43. Lai FY, Nath M, Hamby SE, Thompson JR, Nelson CP, Samani NJ (2018) Adult height and risk of 50 diseases: a combined epidemiological and genetic analysis. BMC Medicine 16(1):187. https://doi.org/10.1186/s12916-018-1175-7

44. Kabat GC, Kim MY, Hollenbeck AR, Rohan TE (2014). Attained height, sex, and risk of cancer at different anatomic sites in the NIH-AARP diet and health study. Cancer Causes Control 25:1697-1706. https://doi.org/10.1007/s10552-014-0476-1

45. Mellemkjaer L, Christense J, Frederiksen K, Baker JL, Olsen A, Sorensen TIA, Tjonneland A (2012) Leg length, sitting height and postmenopausal breast cancer risk. Br J Cancer 107:165-168. https://doi.org/10.1038/bjc.2012.244

46. Fagherazzi G, Viller A, Boutron-Ruault MC, Clavel-Chaperlon F, Mesrine S (2012) Height, sitting height, and leg length in relation with breast cancer risk in the E3N cohort. Cancer Epidemiol Biomarkers Prev 21(7):1171-1175.

https://doi.org/10.1158/1055-9965.EPI-12-0130

47. Ong JS, Ahn J, Law MH, Whiteman DC, Neale RE, Gharahkhani P, MacGregor S (2018) Height and overall cancer risk and mortality: evidence from a Mendelian randomization study on 310,000 UK Biobank participants. Br J Cancer 118:1262-1267. https://doi.org/10.1038/s41416-018-0063-4

Page $16 / 17$ 
48. Keum N, Greenwood DC, Lee DH, Kim R, Aune D, Ju W, Ju FB, Giovannucci EL (2015) Adult weight gain and adiposityrelated cancers: a dose-response meta-analysis of prospective observational studies. J Natl Cancer Inst 107(2): djv088 (pages 1-14). DOI: 10.1093/jnci/djv088

49. John EM, Sangaramoorthy M, Hines LM, Stern MC, Baumgartner KB, Guliano AR, Wolff RL, Slattery ML (2014) Overall and abdominal adiposity and premenopausal breast cancer risk among Hispanic women: The Breast Cancer Health Disparities Study. Cancer Epidemiol Biomarkers Prev 24(1):138-147. https://doi.org/10.1158/1055-9965.EPI-13-1007-T

50. da Silva M, Weiderpass E, Licaj I, Lissner L, Rylander C (2018) Excess body weight, weight gain and obesity-related cancer risk in women in Norway: the Norweigan Women and Cancer study. Br J Cancer 119:646-656. https://doi.org/10.1038/x41416018-0240-5 